\title{
Pierre Vilar : une histoire totale, une histoire en construction
}

Julien Louvrier

\section{(2) OpenEdition \\ 1 Journals}

Édition électronique

URL : https://journals.openedition.org/ahrf/8913

DOI : 10.4000/ahrf.8913

ISSN : 1952-403X

\section{Éditeur :}

Armand Colin, Société des études robespierristes

\section{Édition imprimée}

Date de publication : 1 mars 2007

Pagination : 220-223

ISSN : 0003-4436

\section{Référence électronique}

Julien Louvrier, «Pierre Vilar : une histoire totale, une histoire en construction », Annales historiques de la Révolution française [En ligne], 347 | janvier-mars 2007, mis en ligne le 21 juillet 2008, consulté le 28 juin 2022. URL : http://journals.openedition.org/ahrf/8913 ; DOI : https://doi.org/10.4000/ahrf.8913

Ce document a été généré automatiquement le 23 avril 2022.

Tous droits réservés 


\title{
Pierre Vilar : une histoire totale, une histoire en construction
}

\author{
Julien Louvrier
}

\section{RÉFÉRENCE}

Aron Cohen, Rosa Congost, Pablo F. Luna (dir.), Pierre Vilar : une histoire totale, une histoire en construction, Paris, Syllepse, 2006, 288 p., ISBN 2-84950-097-6, $25 €$.

1 Les articles rassemblés dans cet ouvrage sont le fruit des interventions et des débats tenus dans le cadre de «l'Atelier Pierre Vilar » organisé à Nanterre en octobre 2004, à l'occasion du quatrième Congrès Marx International. Pour les participants à «l'Atelier», il s'agissait d'appliquer à l'œuvre de Pierre Vilar les méthodes et les principes revendiqués par le chercheur lui-même dans sa pratique de l'histoire au quotidien, à savoir exigence de conceptualisation et posture critique. L'intérêt de ce livre réside donc dans la volonté des auteurs de ne pas limiter la réflexion aux louanges, de ne pas résumer la pensée de l'homme à un hommage à l'œuvre.

2 Après une première partie consacrée à l'actualité des thèmes et des questionnements vilariens («Pierre Vilar aujourd'hui »), il est fait état, avec une approche analytique, de l'étendue des intérêts de l'historien, à la fois du point de vue de l'histoire érudite ("L'historien sur le terrain»), et de celui plus théorique des batailles autour de l'histoire économique, du marxisme, de l'histoire sociale (« Analyse historique, analyse des structures des sociétés» et «Histoire et sciences sociales: critiques et construction») ou de la méthode historique («L'historien et l'enseignement de l'histoire »).

3 Pour caractériser en quelques pages la démarche scientifique de Pierre Vilar, Rosa Congost choisit d'insister sur deux formules chères à l'auteur : « histoire raisonnée » et « histoire en construction ». La première est une référence explicite à un texte célèbre de Schumpeter au sujet de Marx, la seconde fait directement allusion au dialogue initié par Vilar avec Althusser. Pour Congost, l'expression utilisée par Schumpeter devient 
pour Pierre Vilar « une sorte de devise, un guide de travail, un modèle jamais achevé ", tandis que l'image utilisée face à Althusser - «ce n'est pas le concept d'histoire, mais l'histoire qu'il faut construire » - est une adaptation d'une parole d'Ernest Labrousse : "l'histoire est à faire ". Aussi n'est-il pas exagéré de dire que c'est par son caractère largement programmatique - «en construction»- que l'histoire vilarienne s'est singularisée. Au cours de sa très longue carrière - il est mort en 2003 à l'âge de 97 ans Vilar ne s'est jamais départi de l'ambition de "tout penser historiquement », plaidant pour une conception de la discipline historique comme un va et vient permanent du cas à la théorie, de la théorie au cas, dont la finalité résiderait dans cette " histoire totale " définie simplement par ces mots : «non pas dire tout sur tout, mais dire ce dont le tout dépend et ce qui dépend du tout ».

4 Ce programme exigeant se retrouve dans le combat mené par Vilar pour une histoire économique quantitative qui ne soit pas simplement "économétrie rétrospective ", selon sa propre expression, une histoire qui ne se réduirait pas à une vulgaire technique et dont il refuse qu'elle se limite à n'être qu'une science auxiliaire au service des sciences économiques. L'attention que porte Pierre Vilar à l'économie et son souci de dialogue équitable entre historiens et économistes font l'objet d'au moins trois communications. Dans la première qui porte sur le rapport présenté au premier Congrès international d'histoire économique de Stockholm en 1960, Claude Mazauric montre comment Vilar, sans rien céder sur ses exigences théoriques et ses positions marxistes, y présente moins un rapport qu'un programme qui, bien que largement adossé à sa thèse dans laquelle il puise les données empiriques de son exposé, contient une charge polémique forte et de portée générale contre les « conceptions idéalistes de la connaissance historique » et la théorie économique néoclassique. Le ralliement des historiens à la démarche des économistes ne peut se faire à n'importe quel prix ! Ce sont en substance les conclusions auxquelles parvient aussi Miriam Halpern : pour Vilar «l'histoire économique ne [doit] en aucun cas s'isoler de l'histoire sociale, de la culture, des mentalités, du politique ».

Dans la troisième communication consacrée à l'histoire économique, Guy Lemarchand examine les différents usages de la notion de mode de production dans l'œuvre de Vilar. Il souligne avec quelle latitude le spécialiste de l'histoire de l'Espagne use de la pertinence d'une notion qui sous sa plume n'est jamais figée, puisqu'il s'agit d'un cadre à la fois « déterminé et déterminant » soumis comme toutes les constructions sociales à l'altérité de son historicité. Dans cette optique, pas question de réduire le travail théorique de Vilar à une conjoncture historique qui serait celle des années soixante, années marquées par la montée en puissance de l'histoire économique et par la forte influence du marxisme dans les sciences sociales. Au contraire, la pensée de Pierre Vilar est tout autant irréductible aux modes passagères, qu'elle serait à l'étroit dans le cadre rigide de "l'historien marxiste" tel que les manuels d'historiographie le conçoivent. Vilar n'a-t-il pas successivement bataillé contre le marxisme politique érigé en dogme dans les années cinquante, puis contre les leçons d'histoire que le philosophe Althusser prétendait, au nom d'une relecture des textes de Marx, adresser aux historiens marxistes (Rosa Congost) ? À dire vrai, le marxisme de Vilar est un marxisme de terrain, sans cesse réévalué et réélaboré au contact de la matière historique.

Ceci étant dit, se pose aujourd'hui la question de la réception de l'œuvre de Pierre Vilar et celle de son devenir. Historien pour historiens, selon le mot d'Eric Hobsbawm, Pierre Vilar n'a jamais bénéficié en France du rayonnement qu'ont connu ses contemporains 
comme Fernand Braudel ou Ernest Labrousse. Outre une carrière universitaire un peu plus tardive, sa thèse monumentale sur la Catalogne (soutenue en 1962 à l'âge de 56 ans) et ses articles théoriques sont restés peu connus du grand public, sinon des cercles spécialisés, historiens marxistes, historiens des révolutions et spécialistes de l'histoire espagnole. Quelle a été son audience dans ces différents milieux, quelle est la place de sa pensée dans l'historiographie contemporaine? Afin de répondre à ces questions il nous faut distinguer deux niveaux d'observation. Le premier niveau doit nous porter à considérer l'inscription de Vilar dans l'historiographie française des années cinquante aux années quatre-vingt, alors qu'il est dans sa pleine maturité intellectuelle, le second nous faire nous interroger sur les tendances de l'historiographie actuelle et le décalage des préoccupations et des intérêts des chercheurs pour le type d'histoire prôné par Vilar.

7 En France, c'est en priorité chez les historiens de la Révolution française qu'on trouvera intérêt et reconnaissance intellectuelle à l'égard des thèses vilariennes. N'oublions pas les liens de Pierre Vilar avec les historiens de la Révolution, en premier lieu avec son maitre Ernest Labrousse auquel il succéda à la chaire d'histoire économique à la Sorbonne, avec son ami Albert Soboul et ses collègues du Centre d'études et de recherches marxistes, dont il est un des membres fondateurs. Dans une conjoncture marquée dès le milieu des années cinquante par la problématique de la transition du féodalisme au capitalisme, telle qu'elle est débattue par l'anglais Dobb, l'américain Sweezy et le japonais Takahashi, la rigueur méthodologique de Pierre Vilar en fait à la fois un guide incontestable et l'historien marxiste auquel il faut se confronter (voir sa participation au recueil Sur le féodalisme, Éditions sociales, Paris, 1971). Il faut noter également que les relations de Pierre Vilar avec les historiens de la Révolution française ne se sont pas limitées aux seules rencontres du CERM, mais qu'il a à plusieurs reprises participé à leurs travaux, donnant des communications à l'occasion de deux colloques majeurs de 1968, celui de Toulouse (L'abolition de la "féodalité» dans le monde occidental, Paris, CNRS, 1971) et celui de Bruxelles (Occupants-Occupés, ULB, 1968). Du côté des spécialistes du monde hispanique, l'influence de Vilar se fait surtout ressentir sur la question nationale (Jacques Maurice, Marcia Mansor d'Alessio), tandis que chez les historiens économiques on retient l'auteur d'une « véritable critique historienne de l'économie politique » (Pablo Luna).

8 Si la liste des contributeurs à ce recueil démontre que Vilar a bien marqué toute une génération d'historiens dont on vient de cerner rapidement les intérêts, on constatera qu'il n'a pas vraiment fait école en France : peu de jeunes chercheurs s'expriment dans ce recueil et peu d'historiens, en dehors des catégories mentionnées, ont cherché à débattre réellement avec lui, et encore moins de spécialistes venus d'autres disciplines. Vilar n'a pourtant jamais cessé de penser les conditions pratiques d'un dialogue, au sens d'un échange critique, de l'histoire avec les sciences sociales, mais ses disciples ne peuvent que constater à quel point il s'est retrouvé seul dans des échanges toujours à sens unique. Quant à ses suggestions portant sur les défaillances du marxisme "réellement existant», on lui a rarement répondu, déplore Pablo Luna. Comment expliquer la solitude de cet historien, célébré en Espagne et en Amérique Latine, mais dont la postérité en France est encore à construire ? Tient-elle justement à sa fidélité au marxisme ? À son exigence d'une histoire "totale »? Pour Rosa Congost, c'est surtout une question de "rupture générationnelle " qui, à partir des années soixante-dix, enferme Vilar dans un moment historiographique, celui des années soixante, dont il serait le produit. Ainsi il apparaîtrait, aux yeux des historiens de la nouvelle génération 
comme une énigme (voir p. 43-44). Mais au-delà de cette distance générationnelle apparaissent aussi un certain nombre de questions, esquissées dans certaines parties du livre, qui peuvent expliquer la méfiance ou la prise de distance vis-à-vis de l'œuvre de Vilar. Les historiens qui ont coordonné l'édition des actes de cette journée ont eu l'excellente intuition de retranscrire les discussions qui ont ponctué le déroulement des séances. C'est au fil de ces pages qu'on lira les arguments les plus critiques à l'égard de la démarche vilarienne. N'a-t-il pas manqué de prendre en considération l'historicité de la société du XIX siècle dans laquelle prend forme la pensée de Marx, demande J. Guilhaumou? N’a-t-il pas sous-évalué les conséquences de l'avènement de la société informationnelle sur l'activité productive, s'interroge Claude Mazauric? Ainsi soumis à l'examen historique, certains aspects de son œuvre ont vieilli, comme ses références appuyées à Staline ou ses affinités intellectuelles avec Mao. Néanmoins, les préceptes méthodologiques sur lesquels il n'a jamais transigé, tels qu'ils s'incarnent dans la formule «tout penser historiquement», constituent pour l'historien d'aujourd'hui et de demain un objectif sûr et ambitieux. 